\title{
Efectos del aprovechamiento forestal en la estructura, diversidad y dinámica de rodales mixtos en la Sierra Juárez de Oaxaca, México
}

\author{
Effects of forestry on the structure, diversity and dynamics of mixed \\ stands in the Sierra Juárez, Oaxaca
}

\author{
Rosario Ramírez Santiago', Gregorio Ángeles Pérez', Patricia Hernández de La Rosa', Víctor Manuel Cetina Alcalá!, \\ Ofelia Plascencia Escalante' y Ricardo Clark-Tapia*
}

1 Colegio de Postgraduados. Postgrado en Ciencias Forestales. Texcoco, Edo. de México, México.

\author{
2 Universidad de La Sierra Juárez. Instituto de estudios \\ Ambientales. Oaxaca, Oaxaca, México. \\ * Autor de correspondencia. rclark@unsij.edu.mx
}

\section{RESUMEN}

Se ha documentado que las prácticas silvícolas en aprovechamientos maderables tienden a simplificar la composición y la complejidad estructural del bosque. Este estudio tiene como objetivo evaluar los cambios en la estructura, la diversidad y la dinámica arbórea de rodales mixtos intervenidos por el método de selección grupal (SG) y árboles padre (AP), así como áreas sin intervenir denominadas bosque de referencia (BR) en la Sierra Juárez de Oaxaca, México. En 2007 y 2012, se obtuvieron datos de diámetro, área basal, altura, supervivencia e incorporación de especies arbóreas de 29 parcelas permanentes de $1000 \mathrm{~m}^{2}$ establecidas en áreas de manejo forestal y conservación. Los resultados mostraron un crecimiento acelerado del arbolado en SG y AP, y ausencia de diferencias en estructura, incremento en volumen y riqueza específica. Sin embargo, BR mostró una distribución diamétrica en forma de "J” invertida, característica de un bosque maduro, así como una mayor diversidad arbórea que los sitios SG y AP. El Incremento medio anual en volumen no mostró diferencias entre las tres condiciones evaluadas al utilizar el total de especies, sin embargo, al analizar solo las especies de pino, SG y AP difirieron con BR, cuyo incremento fue menor. Los resultados generados contribuyen al entendimiento de los procesos ecológicos de los ecosistemas de pino-encino y también a comprender los sistemas silvícolas utilizados y con ello ayudar a formular un manejo sostenible. Se recomienda la aplicación del método de SG que incorpore de manera sistemática la retención de elementos estructurales, para promover la conservación de la biodiversidad en la Sierra de Juárez, Oaxaca.

PALABRAS CLAVE: conservación, estructura forestal, incremento medio anual, parcelas permanentes, sistemas silvícolas.

\section{ABSTRACT}

Silvicultural practices tend to simplify the composition and structural complexity of the forest. The purpose of this study was to assess the changes in forest structure, tree diversity and demographic dynamics of mixed-wood stands harvested by group selection (GS) and seed tree (ST) regeneration methods in the Sierra Juárez of Oaxaca, Mexico. In addition, an unharvested forest was included in the evaluation as a reference site (RF). Data for this study (base area, diameter, height, survival and recruitment) come from 29 permanent sampling plots of $1000 \mathrm{~m}^{2}$ established in the 2007-2012 period. The results revealed that the GS and ST demonstrated rapid growth, despite the high density of the operating areas. In both sites, a similar silvicultural criterion was applied, and this may have been the reason these sites showed no differences in forest structure, annual timber volume increment and species diversity or species richness. However, the GS forest showed features similar to ST, on a wider tree diameter distribution and a higher diversity index for tree species than the RF site. The results generated contribute to the understanding of the ecological processes of the pine-oak ecosystems and to implement the best silvicultural system. Development of intensive forest management that incorporates characteristics of mature forests are recommended as the best practice in terms of forest productivity and ecosystem conservation, in the Sierra Juárez of Oaxaca.

KEYWORDS: conservation, forest structure, mean annual increment, permanent plots, silvicultural system. 


\section{INTRODUCCIÓN}

A escala mundial, los sistemas silvícolas utilizados en los bosques templados para llevar a cabo el aprovechamiento de madera se dividen en dos grandes grupos: sistemas que generan rodales coetáneos y los que generan rodales incoetáneos (Franklin et al., 2002). En México, estos sistemas silvícolas se aplican bajos dos métodos: el Método Mexicano de Ordenación de Bosques Irregulares (MMOBI), que tiene a la corta de selección como principal tratamiento silvícola, ya sea en forma grupal o individual (Solís et al., 2006) y el método de Desarrollo Silvícola (MDS), que está basado en la aplicación de cortas de regeneración a matarrasa o árboles padre, en conjunto con cortas intermedias de aplicación intensiva como los aclareos (Solís et al., 2006).

La estructura, la composición y la diversidad son de los atributos del bosque que más se modifican para alcanzar los objetivos del manejo forestal enfocados en la producción maderable (Franklin et al., 2002; Ishii, Tanabe y Hiura, 2004; Castellanos et al., 2008). El conocimiento sobre la estructura, la diversidad y la dinámica del bosque, así como de los efectos del aprovechamiento forestal sobre estos atributos, es una condición básica para la toma de decisiones sobre el manejo forestal y para mantener la integridad ecológica del mismo (Lutz y Halpern, 2006). Una herramienta útil que permite conocer los efectos del manejo forestal son los sitios permanentes de muestreo (SPM) (Corral, Vargas, Wehenkel, Aguirre y Crecente, 2012).

En México, a diferencia de países como Canadá y Estados Unidos, que desde hace varias décadas han implementado redes de SPM, su uso es relativamente reciente (Corral et al., 2012). En la región de la Sierra Juárez en el estado de Oaxaca, a partir de la década del año 2000 ha sido cada vez más frecuente que se realicen estudios sobre el manejo forestal y sus efectos en la estructura, composición y diversidad en el bosque mixto (p. ej. Castellanos et al., 2008; Ríos-Altamirano et al., 2016). Sin embargo, a excepción de Hernández (2007), ninguno de estos estudios utilizó la medición de sitios permanentes como una fuente de información; por lo que estudios sobre la dinámica y la demografía de rodales han sido escasos.

Capulálpam de Méndez es una comunidad forestal zapoteca localizada en la Sierra Juárez, cuya historia de manejo forestal moderno inició de manera moderada en la pasada década de los 40 y se intensificó a partir de 1956 con la concesión a la empresa Fábricas de Papel Tuxtepec (Fapatux), quien aplicó, inicialmente, el sistema de selección y, posteriormente, el Método Mexicano de Ordenación de Montes (MMOM), basado en la corta selectiva y extracción de los mejores árboles del género Pinus spp. En 1981, año en que terminó la concesión a Fapatux, se estableció el manejo forestal comunitario. En un inicio, la comunidad adoptó el Método Mexicano de Ordenación de Montes Irregulares (MMOBI) y lo sustituyó en 1993 por el Método de Desarrollo Silvícola (MDS), basado en cortas de regeneración de árboles de acuerdo con la Unión de Comunidades Forestales Zapotecas-Chinantecas [Uzachi] (2003). Capulálpam de Méndez es reconocida por ser la primera comunidad en Oaxaca en recibir en 1996 la certificación forestal internacional bajo los principios del Consejo de Administración Forestal (más conocido por sus siglas en inglés como FSC) (Uzachi, 2003); además de ser una las primeras comunidades en la región en implementar sitios permanentes de monitoreo silvicultural (Hernández, 2007). Para lograr una planificación exitosa del manejo forestal, y un conocimiento adecuado de los cambios en la estructura y composición del bosque, los sitios permanentes deben ser monitoreados a través del tiempo, cada cinco años de acuerdo con Hernández (2007).

\section{OBJETIVOS}

El presente estudio tuvo como objetivo evaluar los cambios en la estructura, en la diversidad arbórea y en la dinámica de rodales mixtos bajos diferentes condiciones de manejo forestal en Capulálpam de Méndez, con la finalidad de definir estrategias para el manejo y conservación sustentable de especies arbóreas en la región. 


\section{MATERIALES Y MÉTODOS}

\section{Área de estudio}

El estudio se llevó a cabo en el bosque templado de la comunidad de Calpulálpam de Méndez del municipio del mismo nombre ( $17^{\circ} 18^{\prime} 21^{\prime \prime} \mathrm{N}$ y $\left.96^{\circ} 26^{\prime} 42^{\prime \prime} \mathrm{O}\right)$, dentro de la región de la Sierra Juárez en el estado de Oaxaca (Uzachi, 2003). La altitud varía de $2000 \mathrm{~m}$ a $3080 \mathrm{~m}$ snm (RíosAltamirano et al., 2016). El clima según la clasificación de Köppen, modificado por García (1988), corresponde a C $\left(\mathrm{w}_{2}\right)(\mathrm{w})$ : templado subhúmedo, con régimen de lluvias en verano. La temperatura promedio anual es de $15.2^{\circ} \mathrm{C}$ y la precipitación total anual es de 1115 mm (Hernández, 2007). El bosque templado está compuesto por coníferas y latifoliadas donde los géneros Pinus y Quercus son dominantes, formando diferentes grados de asociación (Uzachi, 2003; Hernández, 2007).

\section{Trabajo de campo}

Los resultados aquí presentados provienen del análisis de dos mediciones efectuadas en el año 2007 y 2012 en 29 sitios permanentes de muestreo (SPM), establecidos en los años 2006 y 2007. Cada SPM es de forma circular con una superficie de $1000 \mathrm{~m}^{2}$, dentro de los cuales solo se consideró arbolado $\geq$ a $10 \mathrm{~cm}$ de diámetro (Hernández, 2007). De cada individuo se tomaron datos de diámetro normal, altura, identificación a nivel de especie y datos demográficos (muerto, vivo e incorporado). Posteriormente se obtuvo el área basal $\left(\mathrm{m}^{2} \mathrm{ha}^{-1}\right)$ y la densidad (individuos ha-1).

Los SPM se encuentran distribuidos en rodales donde se aplicaron de 1993 a 1995 los tratamientos silvícolas de cortas de regeneración de selección grupal (SG) y árboles padre (AP), considerados como silvicultura de baja intensidad y silvicultura intensiva, respectivamente (Hernández, 2007). La silvicultura intensiva (AP) se aplicó en rodales con superficies de cosecha de hasta 2 ha (Hernández, 2007), mientras que el área de silvicultura de baja intensidad (SG) utilizó superficies intervenidas de una hectárea o menor (Uzachi, 2003). En ambos métodos se hicieron plantaciones de pino proveniente de semilla local (Hernández, 2007).

Se establecieron nueve sitios en rodales con SG y seis en rodales con AP. Además, se ubicaron 14 sitios en áreas catalogadas como de conservación y sin manejo forestal por más de tres décadas, a las cuales se les denominó bosque de referencia (BR) (Hernández, 2007). Todas las condiciones forestales (AP, SG y BR) estuvieron localizadas a poca distancia unas de otras $(<1 \mathrm{~km})$ y presentaron condiciones similares de suelo, clima y características geográficas.

\section{Análisis de datos}

El análisis de datos se llevó a cabo considerando todo el conjunto de especies (pinos y latifoliadas), denominado grupo general. Además, se hicieron análisis similares para el grupo de pinos y para el grupo de latifoliadas por separado en las dos fechas de medición. Con la finalidad de comparar estadísticamente los parámetros estructurales se aplicó un análisis de varianza y la prueba no paramétrica de Kruskal Wallis. Para analizar la estructura por tamaños, el intervalo de clase diamétrica fue definida cada $5 \mathrm{~cm}$ y los individuos $\geq 90 \mathrm{~cm}$ se agruparon en la misma clase.

El análisis de las tasas demográficas se efectuó mediante el conteo del número de individuos por hectárea por clase diamétrica. Las categorías de supervivencia se registraron como vivos, muertos e incorporados. Para estimar la tasa de mortalidad (TM) a las clases diamétricas se utilizó la ecuación propuesta por Kubota, Konno y Hiura (1994) [1].

$$
T M=\frac{m i_{2007}}{v i_{2012}-m i_{2007}}
$$

donde:

$v i i_{2012}=$ densidad de individuos vivos en el año 2012

$m i_{2007}=$ individuos muertos en un período de cinco años

La evaluación de la tasa de incorporación (TI) entre los años 2007 y 2012 por clase diamétrica se obtuvo mediante la ecuación planteada por Godínez (2007) [2].

$$
T I=\frac{v i_{2012}+i n_{2012}}{v i_{2007}}
$$


donde:

$v i_{2012}=$ densidad de individuos vivos en el 2012, sin los incorporados en ese año

$v i_{2007}=$ densidad de individuos vivos en el 2007, sin los muertos

$i_{2012}=$ densidad de individuos incorporados en el 2012

Por otra parte, para el cálculo de la tasa de incremento medio anual (IMA), se estimó el volumen de pinos y latifoliadas basándose en el enfoque propuesto por Clark et al. (2001) [3]. Los datos fueron anualizados y extrapolados a la hectárea. Posteriormente, se aplicó una prueba de comparación de medias utilizando LSD de Fisher.

$$
I M A=\frac{\left(\sum\left(\text { vol }_{2012}-\operatorname{vol}_{2007}\right)+\left(\sum\left(\operatorname{vinc}_{2012}-\operatorname{vol}_{a r b 10}\right)\right)\right.}{t_{2}-t_{1}}
$$

donde:

vol ${ }_{\text {año }}=\quad$ volumen de cada individuo medido en el año 2012 y 2007 respectivamente

vinc $2012=$ volumen de los individuos incorporados en el año 2012

vol ${ }_{a r b 10}=$ volumen que tendría un árbol de la misma especie con $10 \mathrm{~cm}$ de diámetro normal (dap)

$t_{2}-t_{1}=\quad$ periodo de medición

La diversidad y riqueza específica se calcularon usando los datos de especies de árboles y su abundancia. Para determinar la riqueza de especies se utilizó el índice de Margalef (Moreno, 2001) y se aplicó la prueba no paramétrica de Kruskal-Wallis para detectar las diferencias entre las condiciones del bosque, en cada año de medición, bajo la hipótesis nula de igualdad de medias y con nivel de significancia de 95\% mediante el programa Infostat versión 2013.

Este índice toma en cuenta el número de especies y el número total de individuos, valores cercanos a cero indican una menor riqueza (Moreno, 2001). Se utilizó además el índice de Shannon-Wiener $\left(\mathrm{H}^{\prime}\right)$ [4] para calcular la diversidad. Este índice aumenta con el número de especies y toma mayores valores cuando las proporciones de las distintas especies son similares (Solís et al., 2006).

$$
H^{\prime}=-\sum p i \ln p i
$$

donde $p i$ es la abundancia proporcional de la especie $i$, lo que implica obtener el número de individuos de la especie $i$ dividido entre el número total de individuos de la muestra.

Después se aplicó la prueba de $t$ modificada de Hutcheson para comparar el índice de diversidad entre las condiciones de manejo evaluadas. Para ello, antes fue necesario calcular la varianza de H'. Una vez calculados los valores de $t$, se procedió a calcular los grados de libertad $(g l)$ para así poder hacer las comparaciones respectivas entre la $t$ calculada y la $t$ de tablas, y determinar posibles diferencias entre las condiciones de manejo del bosque.

\section{Resultados}

\section{Estructura diamétrica}

En ambas fechas de medición, la distribución diamétrica del grupo general del BR mostró una forma de "J" invertida, en la cual la densidad de árboles desciende conforme incrementa la clase diamétrica (Fig. 1). En el grupo de pinos, las primeras cuatro clases diamétricas presentaron proporciones de individuos similares entre sí. Además, en las clases diamétricas de $55 \mathrm{~cm}, 65 \mathrm{~cm}$ y $90 \mathrm{~cm}$ se observó un aumento en la densidad, tanto en el año 2007 como en el año 2012 (Fig. 1). Además, la densidad de pinos en las primeras categorías diamétricas solo representó $12 \%$ de la densidad promedio de este grupo.

En las condiciones bajo manejo forestal (SG y AP) el grupo general presentó en el año 2007 una distribución diamétrica de "J" invertida. En el año 2012, ambas condiciones denotaron una disminución en la densidad de individuos de la clase de $10 \mathrm{~cm}$ y un aumento en la densidad de la clase de $15 \mathrm{~cm}$, que representó 25\% del total de individuos para SG y de 3\% para AP. Por lo que la condición de SG presentó una distribución con tendencia unimodal, aunque AP mantuvo una forma de "J" invertida. Con respecto al grupo de pinos en SG y AP, este presentó una distribución diamétrica de "J" invertida en la primera medición (Fig. 1). En contraste, en el año 2012, la curva 

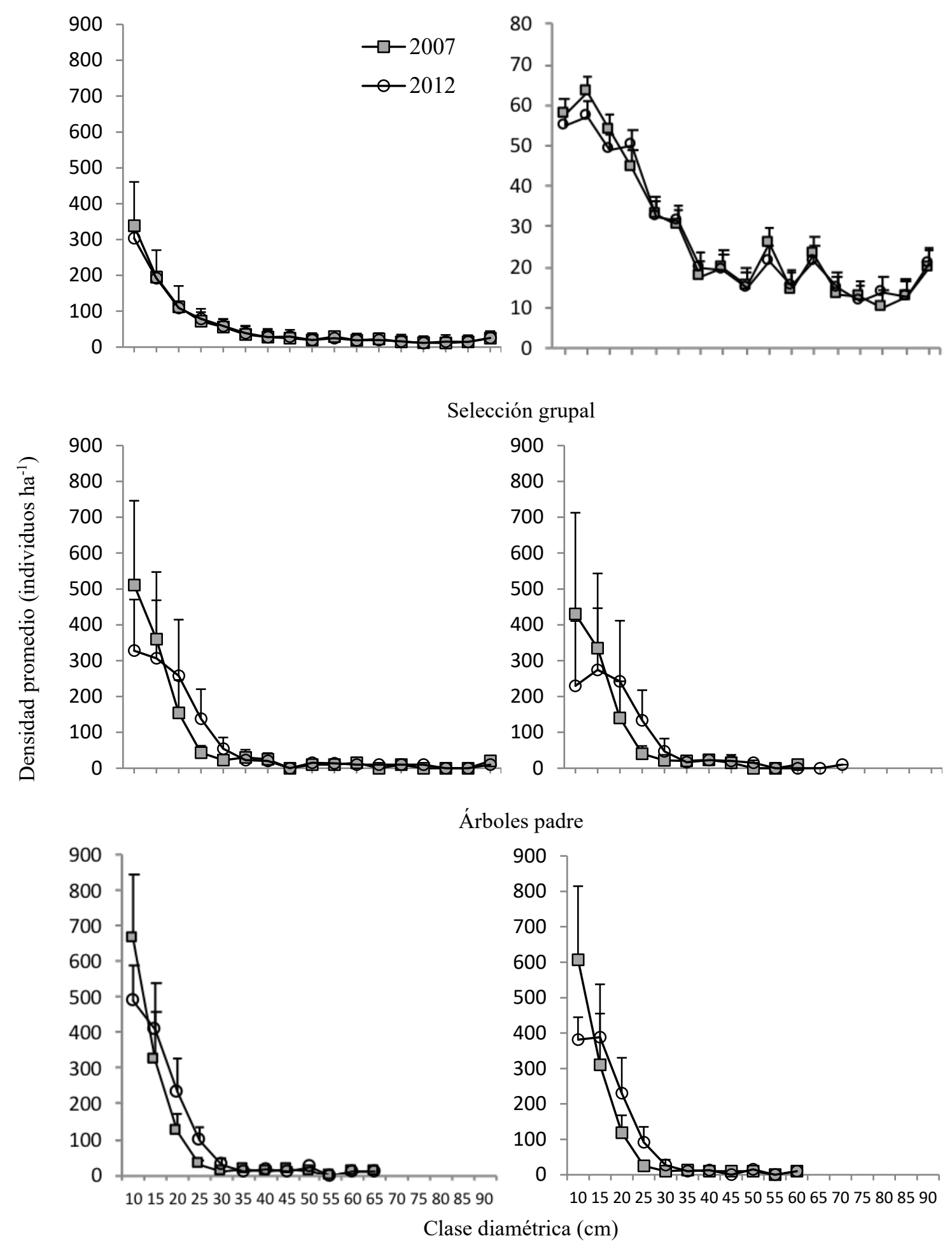

FIGURA 1. Distribución diamétrica total (izquierda) y para el grupo de pinos (derecha) por condición de manejo y por año de medición (individuos con dap $\geq 10 \mathrm{~cm}$ ). En el grupo de pinos del bosque de referencia el eje " $\mathrm{y}$ " es diferente al resto de los paneles. 
adquirió una forma unimodal sesgada a la derecha, con la clase de $15 \mathrm{~cm}$ como la clase moda (Fig. 1).

La prueba de Kruskal-Wallis indicó diferencias significativas entre BR y las condiciones bajo manejo (SG y $\mathrm{AP}$ ) en la mayoría de los parámetros estructurales evaluados (Tabla 1). En todos los casos SG y AP no mostraron diferencias estadísticas entre sí. En ambas condiciones el grupo de pinos fue el dominante en área basal y densidad en los dos periodos de medición. Por el contrario, en el BR el grupo de latifoliadas tuvo la mayor densidad $(76 \%$ del total) con respecto al grupo de pinos (34\% del total), tanto en 2007 como en 2012. Aunque el grupo de pinos conservó más de 60\% del área basal (Tabla 1).

\section{Tasas demográficas}

La tasa de mortalidad (TM) fue menor a uno en todas las clases diamétricas en las que se registró muerte de individuos en las tres condiciones del bosque evaluadas, tanto en el grupo general como en el grupo de pinos. Este valor se considera una tasa de mortalidad baja (Tablas $2 \mathrm{y}$ 3), no obstante, el patrón de mortalidad es diferente en las tres condiciones de manejo evaluadas.

La TM en el grupo general mostró variación entre las categorías diamétricas. En la condición BR la mayor mortalidad se presentó en individuos de la clase diamétricas de $70 \mathrm{~cm}$. En SG las clases más afectadas fueron la de 40 $\mathrm{cm}$ y $>90 \mathrm{~cm}$, mientras que en AP la clase menor a $15 \mathrm{~cm}$ fue la más afectada, en todos los casos la mortalidad fue más contundente en el grupo de pinos.

En el BR la tasa de incorporación (TI) del grupo general no mostró cambios importantes en la densidad de las clases diamétricas, aunque TI elevadas $(>1)$ se presentaron en las clases de $45 \mathrm{~cm}, 70 \mathrm{~cm}$ y $75 \mathrm{~cm}$ (Tabla 2), evidenciado un aumento en la densidad de árboles de mayor tamaño. Algo similar sucedió en la TI del grupo de pinos para las mismas clases de tamaño (Tabla 3).

A su vez, en el grupo general y el grupo de pinos de SG y AP, la TI fue $<1$ en las clases diamétricas más pequeñas (Tablas 2 y 3), lo que significa una disminución en la densidad de individuos o una escasa incorporación de nuevos individuos. Por el contrario, se encontraron valores elevados en TI en las clases diamétricas intermedias $(20,25$, 30 y 35), lo que fue todavía más evidente en el grupo de pinos (Tablas 2 y 3). Se registró que una gran cantidad de individuos presentaron un crecimiento acelerado que les permitió trasladarse a las siguientes categorías en el período de tiempo evaluado (5 años).

TABLA 1. Descripción de la estructura del rodal por condición y año de medición ( $\mathrm{dbh} \geq 10 \mathrm{~cm})$ en rodales mixtos de la Sierra Juárez de Oaxaca, México.

\begin{tabular}{|c|c|c|c|c|c|c|c|c|c|c|}
\hline \multirow{2}{*}{ Año } & \multirow{2}{*}{ Variable } & \multicolumn{3}{|c|}{ Pinos } & \multicolumn{3}{|c|}{ Latifoliadas } & \multicolumn{3}{|c|}{ Grupo general } \\
\hline & & $B R^{*}$ & $S G^{*}$ & $A P^{*}$ & $B R$ & SG & $A P$ & $B R$ & SG & $A P$ \\
\hline \multirow{4}{*}{2007} & Densidad (individuos ha-1) & $317 a$ & $973 b$ & $1068 b$ & $644 b$ & $156 a$ & $78 a$ & $961 a$ & $1129 a$ & $1147 a$ \\
\hline & Dap promedio (cm) & $43.5 b$ & $18.04 a$ & $15.50 a$ & 19.90a & $18.89 a$ & $16.04 a$ & $25.81 b$ & $18.02 a$ & $15.53 a$ \\
\hline & Área basal $\left(\mathrm{m}^{2} \mathrm{ha}^{-1}\right)$ & $44.15 a$ & $27.63 a$ & $38.27 a$ & $26.9 b$ & $10.50 a$ & $2.14 \mathrm{a}$ & $71.13 \mathrm{~b}$ & 38.13 a & $40.41 \mathrm{a}$ \\
\hline & Número de especies & 6 & 4 & 5 & 42 & 16 & 8 & 48 & 20 & 13 \\
\hline \multirow{4}{*}{2012} & Densidad (individuos ha-1) & $311 \mathrm{a}$ & $959 b$ & $1127 b$ & $634 \mathrm{~b}$ & $178 a$ & $143 a$ & $945 a$ & $1137 a b$ & $1270 b$ \\
\hline & Dap promedio $(\mathrm{cm})$ & $46.27 \mathrm{~b}$ & $21.72 a$ & $18.11 a$ & $20.8 b$ & $18.4 \mathrm{ab}$ & $14.4 a$ & $27.15 b$ & $20.79 a$ & $17.77 a$ \\
\hline & Área basal $\left(\mathrm{m}^{2} \mathrm{ha}^{-1}\right)$ & $50.50 \mathrm{a}$ & $35.50 a$ & $32.41 \mathrm{a}$ & $30.76 \mathrm{~b}$ & $8.58 \mathrm{a}$ & $3.57 \mathrm{a}$ & $81.26 \mathrm{~b}$ & $44.08 \mathrm{a}$ & $35.98 \mathrm{a}$ \\
\hline & Número de especies & 6 & 4 & 5 & 42 & 16 & 10 & 48 & 21 & 16 \\
\hline
\end{tabular}

*BR= Bosque de referencia, $S G=$ Selección grupal; $A P=$ Árboles padre. Letras distintas dentro de las columnas indican diferencias estadísticas significativas entre condiciones de manejo ( $P \leq \mathrm{O} .05)$. 
TABLA 2. Tasas de mortalidad e incorporación, por clase de tamaño para el BR, SG y AP para todo el conjunto de especies de rodales mixtos de la Sierra Juárez de Oaxaca, México.

\begin{tabular}{|c|c|c|c|c|c|c|}
\hline \multirow[b]{2}{*}{$\begin{array}{l}\text { Clase } \\
\text { diamétrica }\end{array}$} & \multicolumn{2}{|c|}{ Bosque de referencia (BR) } & \multicolumn{2}{|c|}{ Selección grupal (SG) } & \multicolumn{2}{|c|}{ Arboles padre (AP) } \\
\hline & $\begin{array}{l}\text { Tasa de } \\
\text { mortalidad } \\
\text { (TM) }\end{array}$ & $\begin{array}{c}\text { Tasa de } \\
\text { incorporación } \\
\text { (TI) }\end{array}$ & $\begin{array}{l}\text { Tasa de } \\
\text { mortalidad } \\
\text { (TM) }\end{array}$ & $\begin{array}{c}\text { Tasa de } \\
\text { incorporación } \\
\text { (TI) }\end{array}$ & $\begin{array}{l}\text { Tasa de } \\
\text { mortalidad } \\
\text { (TM) }\end{array}$ & $\begin{array}{c}\text { Tasa de } \\
\text { incorporación } \\
\text { (TI) }\end{array}$ \\
\hline $10-14.99$ & 0.059 & 0.948 & 0.103 & 0.683 & 0.115 & 0.790 \\
\hline 15-19.99 & 0.047 & 1.023 & 0.080 & 0.879 & 0.039 & 1.295 \\
\hline $20-24.99$ & 0.063 & 1.034 & 0.073 & 1.699 & 0.045 & 1.931 \\
\hline $25-29.99$ & 0.013 & 1.100 & 0.067 & 3.263 & 0.000 & 5.182 \\
\hline $30-34.99$ & 0.019 & 1.052 & 0.125 & 2.882 & - & 6.000 \\
\hline 35-39.99 & 0.000 & 1.102 & 0.000 & 1.333 & 0.000 & 0.750 \\
\hline $40-44.99$ & 0.000 & 1.083 & 0.333 & 1.111 & 0.000 & 2.000 \\
\hline $45-49.99$ & 0.045 & 1.462 & 0.000 & 2.250 & - & 0.333 \\
\hline $50-54.99$ & 0.000 & 1.000 & - & 1.500 & 0.000 & 4.000 \\
\hline 55-59.99 & 0.045 & 0.964 & 0.000 & 2.000 & - & - \\
\hline $60-64.99$ & 0.000 & 0.905 & 0.000 & 0.333 & 0.000 & 1.000 \\
\hline $65-69.99$ & 0.077 & 0.833 & - & - & 0.000 & 1.000 \\
\hline 70-74.99 & 0.143 & 1.778 & - & 1.000 & - & - \\
\hline 75-79.99 & 0.000 & 1.600 & - & - & - & - \\
\hline $80-84.99$ & 0.000 & 1.000 & - & - & - & - \\
\hline 85-89.99 & 0.000 & 1.000 & - & 0.000 & - & - \\
\hline$\geq 90$ & 0.000 & 1.214 & 0.526 & 1.000 & - & - \\
\hline Total & 0.046 & 1.022 & 0.095 & 1.052 & 0.090 & 1.156 \\
\hline
\end{tabular}

TABLA 3. Tasa de mortalidad y de incorporación por clase de tamaño para el grupo de pinos del BR, SG y AP en de rodales mixtos de la Sierra Juárez de Oaxaca. México.

\begin{tabular}{|c|c|c|c|c|c|c|}
\hline \multirow[b]{2}{*}{$\begin{array}{l}\text { Clase } \\
\text { diamétrica }\end{array}$} & \multicolumn{2}{|c|}{ Bosque de referencia (BR) } & \multicolumn{2}{|c|}{ Selección grupal (SG) } & \multicolumn{2}{|c|}{ Arboles padre (AP) } \\
\hline & $\begin{array}{l}\text { Tasa de } \\
\text { mortalidad } \\
\text { (TM) }\end{array}$ & $\begin{array}{c}\text { Tasa de } \\
\text { incorporación } \\
\text { (TI) }\end{array}$ & $\begin{array}{l}\text { Tasa de } \\
\text { mortalidad } \\
\text { (TM) }\end{array}$ & $\begin{array}{c}\text { Tasa de } \\
\text { incorporación } \\
\text { (TI) }\end{array}$ & $\begin{array}{l}\text { Tasa de } \\
\text { mortalidad } \\
\text { (TM) }\end{array}$ & $\begin{array}{c}\text { Tasa de } \\
\text { incorporación } \\
\text { (TI) }\end{array}$ \\
\hline $10-14.99$ & 0.054 & 0.827 & 0.120 & 0.572 & 0.131 & 0.676 \\
\hline 15-19.99 & 0.017 & 0.919 & 0.087 & 0.846 & 0.043 & 1.274 \\
\hline $20-24.99$ & 0.018 & 0.931 & 0.069 & 1.758 & 0.048 & 1.971 \\
\hline $25-29.99$ & 0.018 & 1.146 & 0.083 & 3.871 & 0.000 & 5.500 \\
\hline $30-34.99$ & 0.028 & 1.125 & 0.000 & 3.231 & - & 9.000 \\
\hline 35-39.99 & 0.000 & 0.917 & 0.000 & 1.667 & 0.000 & 0.500 \\
\hline 40-44.99 & 0.000 & 1.048 & 0.000 & 1.286 & 0.000 & 4.000 \\
\hline 45-49.99 & 0.043 & 1.533 & - & 2.000 & - & 0.000 \\
\hline $50-54.99$ & 0.000 & 1.091 & - & - & 0.000 & 3.000 \\
\hline $55-59.99$ & 0.000 & 0.833 & - & - & - & - \\
\hline $60-64.99$ & 0.000 & 0.875 & - & 0.000 & 0.000 & 1.000 \\
\hline 65-69.99 & 0.058 & 0.850 & - & - & - & - \\
\hline 70-74.99 & 0.081 & 1.714 & - & - & - & - \\
\hline 75-79.99 & 0.000 & 1.400 & - & - & - & - \\
\hline $80-84.99$ & 0.000 & 1.167 & - & - & - & - \\
\hline 85-89.99 & 0.000 & 1.000 & - & - & - & - \\
\hline$\geq 90$ & 0.000 & 1.228 & - & - & - & - \\
\hline Total & 0.023 & 1.005 & 0.103 & 1.029 & 0.100 & 1.105 \\
\hline
\end{tabular}




\section{Incremento medio anual en volumen (IMA)}

De acuerdo con la prueba de comparación de LSD aplicada no se detectaron diferencias significativas entre BR (18.13 $\mathrm{m}^{3} \mathrm{ha}^{-1}$ año-1 $)$, SG $\left(24.62 \mathrm{~m}^{3} \mathrm{ha}^{-1}\right.$ año $\left.^{-1}\right)$ y AP $\left(18.73 \mathrm{~m}^{3} \mathrm{ha}^{-1}\right.$ año-1) para el incremento medio anual en volumen (IMA), considerando todas las especies de manera conjunta (grupo general).

En cambio, se observaron diferencias significativas $(P$ $=0.0125)$ en el IMA en volumen para el grupo de especies de pino entre condiciones evaluadas de acuerdo con la prueba de LSD. El IMA para SG fue mayor $\left(21.85 \mathrm{~m}^{3} \mathrm{ha}^{-1}\right.$ año-1) que el IMA de BR (13.01 $\mathrm{m}^{3}$ ha- $^{-1}$ año-1 $^{-1}$. No hubo diferencias estadísticas significativas entre el IMA de SG y el de AP $\left(17.33 \mathrm{~m}^{3} \mathrm{ha}^{-1}\right.$ año-1).

\section{Riqueza específica y diversidad arbórea}

Con relación a la riqueza específica, la prueba de Kruskal Wallis detectó diferencias significativas entre BR y la condiciones bajo manejo forestal en el año 2007 ( $\mathrm{H}=$ 11.58; $P \leq 0.0028)$ y de igual manera en el año $2012(\mathrm{H}=$ 8.75; $P \leq 0.0121)$, debido a que posee un mayor número de especies registradas en comparación con SG y AP (cuadro 4). Entre estas últimas no hubo diferencias estadísticas significativas para ambos periodos de medición (Tabla 4).

En cuanto al índice de diversidad de Shannon, las tres condiciones del bosque presentaron diferencias significativas entre sí (Tabla 4), en ambos periodos de medición, como lo reveló la prueba de $t$ de Hutchenson. El BR presenta el mayor valor del índice de diversidad, seguido de la condición de SG y por último la condición de AP (Tabla 4). Los valores más elevados de H' en BR son la consecuencia de un número mayor de especies y con una abundancia similar. Por el contrario, en las condiciones de SG y AP, la dominancia y mayor abundancia son para Pinus, que es el género de mayor interés comercial en el área de estudio. El hecho de que SG obtuviera un valor de diversidad más alto que AP estuvo relacionada a una mayor presencia y abundancia de especies de latifoliadas.

\section{DISCUSIÓN}

\section{Estructura diamétrica}

La diferencia estructural encontrada entre rodales manejados (SG y AP) y no manejados (BR) es consistente con la estructura documentada por diversos autores como Ishii et al. (2004), Hernández et al., (2013) y Clark-Tapia et al. (2017), en bosques templados. La distribución diamétrica de " $\mathrm{J}$ " invertida obtenida en BR para ambos períodos de medición es debida a una mayor densidad de especies de latifoliadas, que sugiere una regeneración favorable y un sistema maduro en crecimiento (Lähde, Eskelinen y Väänänen, 2002). Estas condiciones han sido generadas por un aprovechamiento de tipo selectivo de hace 50 años y por disturbios menores (p. ej. la caída de árboles individuales) que ha propiciado un escaso reclutamiento de pinos, similar a lo que lo encontrado por Fraver y Palik (2012) para Pinus resinosa. Otra diferencia notable del BR con relación a SG y AP es una mayor densidad y área basal del grupo de latifoliadas, permitiendo que se desarrolle una estructura más compleja (Ishii et. al., 2004). Condiciones similares han sido documentadas por Castellanos et al. (2008) en una localidad cercana al área de estudio; estos autores mencionan que conforme el bosque se desarrolla en etapas sucesionales más avanzadas, las latifoliadas tienen una tendencia a aumentar su densidad y dominancia con respecto a las especies de pino, que coincide con lo obtenido en el presente trabajo.

En contraste, la estructura de las condiciones de SG y AP mostró un cambio de distribución de una forma de "J" invertida, a una con tendencia unimodal sesgada a la derecha, siendo más evidente para el grupo de especies de pinos. Este cambio entre periodos de medición en la forma de la distribución diamétrica ha sido observado por Návar y Domínguez (2013) en bosques templados del norte de México, y se ha atribuido a la presencia de árboles en clases de tamaño pequeño e intolerantes a la sombra (Návar y Domínguez, 2013; Silver, D’Amato, Fraver, Palik y Bradford, 2013). 
TABLA 4. Riqueza específica (S) y diversidad de árboles $\geq 10 \mathrm{~cm}$ dap $\left(\mathrm{H}^{\prime}\right)$ en rodales mixtos de la Sierra Juárez de Oaxaca, México.

\begin{tabular}{ccccc}
\hline \multirow{2}{*}{ Condición } & \multicolumn{2}{c}{ Riqueza específica } & \multicolumn{2}{c}{ Shannon-Wiener } \\
\cline { 2 - 5 } & S2007 & S2012 & $H^{\prime} 2007$ & $H^{\prime} 2012$ \\
\hline BR $^{*}$ & $12 \mathrm{~b}$ & $12 \mathrm{~b}$ & $2.77 \mathrm{c}$ & $2.82 \mathrm{c}$ \\
\hline $\mathrm{SG}^{*}$ & $7 \mathrm{a}$ & $8 \mathrm{a}$ & $1.18 \mathrm{~b}$ & $1.25 \mathrm{~b}$ \\
\hline $\mathrm{AP}^{*}$ & $4 \mathrm{a}$ & $6 \mathrm{a}$ & $0.81 \mathrm{a}$ & $1.05 \mathrm{a}$ \\
\hline
\end{tabular}

*BR = Bosque de referencia; SG = Selección grupal; AP = Árboles padre. Letras distintas dentro de las columnas indican diferencias estadísticas significativas entre condiciones de manejo $(P \leq 0.05)$.

Un aspecto a destacar en los atributos estructurales evaluados fue la similitud entre los tratamientos silvícolas SG y AP, que contradijo la hipótesis de este estudio. Se esperaba encontrar una respuesta diferente de los parámetros estructurales entre los métodos de regeneración empleados, al tratarse de sistemas silvícolas distintos. Además, en ambas condiciones de estudio se han generado masas con una elevada densidad de árboles debido a la ausencia de aprovechamiento y a la aplicación de aclareos de baja intensidad (Hernández, 2007) que han removido un área basal menor a 5\%. El aumento en la dominancia del grupo de pinos con respecto al grupo de latifoliadas es una característica común encontrada en áreas bajo manejo forestal (Solís et al., 2006; Hernández et al., 2013); debido a la tendencia a simplificar la composición del rodal intervenido, para generar una masa forestal coetánea acorde al objetivo primordial de producción de madera (Puettman et al., 2009; Fraver y Palik, 2012).

\section{Tasas demográficas}

A pesar de la densidad elevada que presentaron las condiciones evaluadas, las tasas de incorporación en las tres condiciones sugieren un proceso de crecimiento acelerado. Esta respuesta está relacionada con las condiciones de alta productividad (condiciones climáticas y fertilidad de los suelos) presente en la región de estudio que permite el desarrollo de la vegetación como lo señalan Clark-Tapia et al. (2017).

En el caso de la condición BR, la dinámica forestal responde a patrones de incorporación y mortalidad característicos de bosques en etapa madura, adecuada para el desarrollo de especies tolerantes a la sombra (Fraver y Palik, 2012) como las latifoliadas, mas no para especies como los pinos que son generalmente intolerantes a la sombra (Silver et al., 2013); esto podría explicar la tasa de incorporación baja de este grupo en clases diamétricas pequeñas, lo que resulta en una menor dominancia respecto al grupo de latifoliadas. Por el contrario, cuando se brindan las condiciones de mantenimiento (aclareos o tratamientos de liberación) del rodal manejado, la incorporación de pinos tiende a incrementarse como se registró en este estudio en SG y AP, a pesar de que se aplicaron aclareos de baja intensidad (Hernández, 2007).

Por otra parte, la mortalidad observada en este estudio en BR tuvo un patrón similar al mencionado por Vilá, Martínez, Vayreda y Retana (2011) en un bosque no manejado de Pinus sylvestris (L.) en donde la mortalidad afectó tanto a árboles pequeños como a árboles grandes. Este patrón ocurre cuando la competencia deja de ser el factor más relevante de mortalidad en bosques en etapa madura (Das et al., 2011) y las causas de mortalidad dependen más de variables abióticas (Das, Battles, Stephenson y van Mantgem, 2011). En el área de estudio no se puede descartar la competencia inter e intraespecífica como factor de mortalidad en las tres condiciones estudiadas, ni dar mayor peso a las variables abióticas, por lo que es un aspecto que deberá ser estudiado a futuro para comprender los procesos ecológicos de los ecosistemas de pino-encino, que regulan el comportamiento demográfico de un rodal. 


\section{Incremento medio anual en volumen (IMA)}

La ausencia de diferencias significativas entre las tres condiciones evaluadas en el IMA del total de especies no solo descarta la hipótesis de este estudio, sino que contrasta lo señalado por Rossi, Tremblay, Morin y Savard (2009), quienes mencionan un mayor crecimiento en estructuras coetáneas (en este caso SG y AP) que en estructuras incoetáneas (BR) por efecto de un lento crecimiento en los árboles como resultado de una supresión inicial por la competencia con la capa dominante. Sin embargo, coinciden con lo encontrado por Lähde et al. (2002) en donde el IMA fue mayor en un bosque maduro que en bosques bajo manejo. Aun cuando es necesario realizar más investigaciones, esto puede estar relacionado con la complejidad estructural del rodal que promueve una utilización complementaria de los recursos existentes, lo que aumenta su productividad; resultado hallado también por Ishii et al. (2004).

Considerando que el IMA del grupo de pinos el rodal de SG no difiere de AP, de acuerdo con Valappil et al. (1997), los rodales manejados con el sistema de selección pueden mantener niveles comparables de productividad de madera con los rodales manejados por sistemas coetáneos, como lo manifestaron los resultados del estudio.

\section{Riqueza específica y diversidad arbórea}

Los resultados en este apartado avalan la hipótesis del presente estudio, de que hay una mayor riqueza y diversidad en la condición de BR. Los resultados encontrados en SG y AP muestran una consecuencia bien documentada del manejo forestal, encaminado a simplificar un rodal para fomentar la dominancia de una sola especie (Solís et al., 2006; Hernández et al., 2013; Clark-Tapia et al., 2017). Además, Castellanos et al. (2008) mencionan que bosques maduros, como el BR de este estudio, contienen un mayor número de especies que aquellos que fueron intervenidos recientemente y se encuentran en una etapa de desarrollo juvenil. De acuerdo con Franklin et al. (2002) cuando operan disturbios a pequeña escala se promueve la diversidad alfa, lo que permite el aumento en la presencia de especies tolerantes a la sombra, y con ello se incrementa la riqueza de especies e índices de diversidad como el de Shannon-Wiener.

Los resultados de este estudio concuerdan con lo encontrado por Solís et al. (2006) y Hernández et al. (2013), quienes han registrado una disminución de la diversidad arbórea en bosques templados de México bajo intervención silvícola, donde las cortas de selección y de árboles padre fueron aplicadas. Así mismo, estos valores coinciden con lo hallado por Castellanos et al. (2008) y Clark-Tapia et al. (2017) en bosques templados bajo manejo forestal dentro del estado de Oaxaca. No obstante, es importante señalar que SG tuvo un valor más alto de diversidad con respecto a AP, lo que apoyó la hipótesis de que las condiciones bajo manejo forestal son diferentes entre sí, y ello se debe a la diferencia entre los tratamientos implementados en Capulálpam de Méndez (Hernández, 2007).

\section{Implicaciones para el manejo forestal local}

Los resultados generados contribuyen no solo al entendimiento de los procesos ecológicos de los ecosistemas de pino-encino, sino también a comprender los sistemas de tratamiento utilizados y con ello, ayudar a formular un manejo sustentable local. El método de SG posee ventajas frentes a métodos coetáneos, ya que permite agregar diversidad estructural al generar pequeños espacios coetáneos, lo cual favorece la diversidad biológica, mantiene una distribución diamétrica más amplia y una cobertura forestal continua. Esto produce rodales que se aproximan más a un régimen de disturbio natural, con una mayor calidad estética y diversidad estructural como ha sido documentado en diversos estudios como los de Franklin et al. (2002) y Lähde et al. (2002).

Por el contrario, los sistemas coetáneos simplifican la estructura del rodal al reducir la variabilidad del tamaño de los individuos arbóreos y disminuyen la diversidad de especies, entre otros componentes, disminuyendo las funciones del ecosistema (Ishii et al., 2004). Para Franklin et al. (2002) y Rossi et al. (2009), estos sistemas tienen poco en común con los disturbios naturales de reemplazo de rodal, 
como comúnmente se cree, excepto por crear condiciones más adecuadas de luz para la regeneración de especies intolerantes (Franklin et al., 2002). Sin embargo, es necesario realizar estudios futuros que incluyan los efectos de otros tratamientos silvícolas no evaluados en este estudio (p. ej. matarrasa en franjas alternas), así como evaluar otros componentes y procesos del bosque con manejo y sin manejo. Esto con la finalidad de mejorar la aplicación de los tratamientos silvícolas o la implementación de acciones de conservación; y de esta manera cumplir con los objetivos económicos, productivos y ecológicos que deben considerarse como parte de un manejo forestal sostenible. Para ello, es importante continuar con la medición periódica de la red de sitios permanentes de muestreo con la que cuenta la comunidad de Capulálpam de Méndez.

\section{CONCLUSIONES}

El uso de parcelas permanentes fue una herramienta bastante útil para monitorear a través del tiempo el cambio en la estructura, tasas demográficas, incremento en volumen y diversidad arbórea del bosque en la comunidad de Capulálpam de Méndez. La estructura diamétrica mostró cambios en el tiempo, en 2007 las condiciones estudiadas (BR, SG y AP) mostraron una estructura diamétrica en forma de "J" invertida, mientras que, en 2012 con excepción de $\mathrm{BR}$, presentaron una distribución con tendencia unimodal. En el período de estudio, la tasa de mortalidad fue menor a uno en todas las clases diamétricas en las que se registró muerte de individuos, tanto en el grupo general como en el grupo de pinos. El análisis del incremento medio anual en volumen (IMA), no mostró diferencias entre BR, SG y AP al considerarse a todas las especies analizadas de manera conjunta, sin embargo, sí las hubo entre condiciones cuando se evaluó de manera exclusiva al grupo de coníferas. Finalmente, el uso de parcelas permanentes permite detectar diferencias en la diversidad de especies entre BR y las condiciones bajo manejo forestal. Con base a los resultados de este estudio, para promover la conservación de la biodiversidad, se sugiere incorporar a los planes de manejo forestal, la retención de elementos estructurales conformada por especies de latifoliadas, además de las coníferas. Estos elementos no solo pueden servir para mejorar la aplicación del MMOBI (SG), sino también para mejorar los resultados del MDS (AP) en Capulálpam de Méndez.

\section{RECONOCIMIENTOS}

Los autores agradecen el apoyo del Programa Ciencia Básica SEP Conacyt No. 180790. También agradecemos al Comisariado de Bienes Comunales de Capulalpam de Méndez (periodo 2010-2013) y a la Dirección Técnica Forestal por su interés y apoyo en la ejecución de este estudio. La primera autora de este trabajo agradece a los estudiantes de la Universidad de la Sierra Juárez de Oaxaca que colaboraron en el trabajo de campo.

\section{REFERENCIAS}

Castellanos, J. F., Treviño, J. E., Aguirre, O. A., Jiménez, J., Musalem, M., \& López, R. (2008). Estructura de bosques de Pinus patula bajo manejo en Ixtlán de Juárez, Oaxaca, México. Madera y Bosques, 14(2), 51-63. doi: 10.21829/myb.2008.1421212

Clark, A. D., Brown, S., Kicklighter, D. W., Chambers, J. Q., Thomlinson, J. R., \& Ni, J. (2001). Measuring net primary production in forests: concepts and field methods. Ecological Applications, 11(2), 356-370. doi: 10.2307/3060894

Clark-Tapia, R., Aguirre-Hidalgo, V., Alfonso-Corrado, C., Ramírez, R. S., Domínguez, R. Y., Martínez, P. A., Aquino, C. V., Jiménez, L. B., Aparicio, M. G., Pacheco, P. R., \& Sarmiento, D. B. (2017). Estructura, composición y diversidad de la vegetación en areas de manejo forestal de Ixtlán de Juárez, Oaxaca. En R. Clark-Tapia, M. C. Fuente, C. Alfonso-Corrado, F. M. Ramos, \& V. Aguirre-Hidalgo (Coords.). Manejo Forestal Comunitario y Sustentabilidad en Sierra Juárez, Oaxaca (pp. 58-77). México: Fontamara.

Corral, J. J., Vargas, B., Wehenkel, C., Aguirre, O. A., \& Crecente, F. (2012). Guía para el establecimiento, seguimiento y evaluación de sitios permanentes de monitoreo en paisajes productivos forestales. México: Comisión Nacional Forestal-Comisión Nacional de Ciencia y Tecnología.

Das, A., Battles, J., Stephenson, N. L., \& van Mantgem, P. J. (2011). The contribution of competition to tree mortality in old-growth coniferous forests. Forest Ecology and Management, 261(7), 1203-1213. doi: 10.1016/j.foreco.2010.12.035

Franklin, J. F., Spies, T. A., Van Pelt, R., Carey, A. B., Thornburgh, D. A., Berg, D. R., Lindenmayer, D. B., Harmon, M. E., Keaton, W. S., Shaw, D. C., Bible, K., \& 
Chen, J. (2002). Disturbances and structural development of natural forest ecosystems with silvicultural implications, using Douglas-fir forests as an example. Forest Ecology and Management, 155(1-3), 399-423.

Fraver, S. \& Palik, B. J. (2012). Stand and cohort structures of old-growth Pinus resinosa dominated forests of northern Minnesota, USA. Journal of Vegetation Science, 23(2), 249-259. doi: 10.1111/j.1654-1103.2011.01348.x

García, E. (1988). Modificaciones al sistema de clasificación climática de Köppen. (Para adaptarlo a las condiciones de la República Mexicana). D.F., México: Offset Larios.

Godínez, I. O. (2007). Dinámica de la regeneración de Fagus grandifolia Ehrh. subsp. mexicana (Martínez) E. Murray en La Mojonera, Zacualtipán, Hidalgo. Tesis de doctorado. Colegio de Postgraduados, Montecillo, Texcoco, Estado de México.

Hernández, L. I. (2007). Cambios en la estructura y composición del bosque bajo dos tratamientos silviculturales en la Comunidad de Capulálpam de Ménder, Ixtlán, Oaxaca, México. Tesis de maestría. CATIE, Turrialba, Costa Rica.

Hernández, J., Aguirre, O. A., Alanís, E., Jiménez, J., Treviño, E. J., González, M. A., Luján, C., Olivas, J. M., \& Domínguez, L. A. (2013). Efecto del manejo forestal en la diversidad y composición arbórea de un bosque templado del noroeste de México. Revista Chapingo Serie Ciencias Forestales y Del Ambiente, 19, 189-199. doi: 10.21829/myb.2018.2421767

Ishii, H. T., Tanabe, S., \& Hiura, T. (2004). Exploring the relationships among canopy structure, stand productivity, and biodiversity of temperate forest ecosystems. Forest Science, 50(3), 342-355. doi: 10.1093/forestscience/50.3.342

Kubota, Y., Konno, Y., \& Hiura, T. (1994). Stand structure and growth patterns of understory trees in a coniferous forest, Taisetsuzan National Park northern Japan. Ecological Research, 9(3), 333-341. doi: 10.1007/BF02348420

Lähde, T. E., Eskelinen, T., \& Väänänen A. (2002). Growth and diversity effects of silvicultural alternatives on an old-growth forest in Finland. Forestry, 75(4), 395-400. doi: 10.1093 / forestry/75.4.395

Lutz, J. A., \& Halpern, B. Ch. (2006). Tree mortality during early forest development: a long-term study of rates, causes and consequences. Ecological Monographs, 76(2), 257-275. doi: 10.1890/0012-9615(2006)076[0257:TMDEFD]2.0.CO;2

Moreno, C. (2001). Métodos para medir la biodiversidad. M\&TManuales y Tesis SEA, vol. 1. Zaragoza, España.

Návar, J. J., \& Domínguez, A. (2013). Modelo de incremento y rendimiento: ejemplos y aplicaciones para bosques templados mexicano. Revista Mexicana Ciencias Forestales, 4(18), 8-26.
Ríos-Altamirano, A., Alfonso-Corrado, C., Aguirre-Hidalgo, V., Ángeles-Pérez, G., Mendoza-Díaz, M., Rodríguez-Rivera, V., Roldán-Felix, E., \& Clark-Tapia, R. (2016). Abundancia y distribución del género Pinus en Capulálpam de Méndez, Sierra Juárez, Oaxaca. Madera y Bosques, 22(3), 61-74. doi: $10.21829 /$ myb.2016.2231457

Rossi, S., Tremblay, M. J., Morin, H., \& Savard, G. (2009). Growth and productivity of black spruce in even- and uneven-aged stands at the limit of the closed boreal forest. Forest Ecology and Management, 258(9), 2153-2161. doi: 10.1016/j.foreco.2009.08.023

Silver, E., D’Amato, A. W., Fraver, S., Palik, B. J., \& Bradford, J. B. (2013). Structure and development of old-growth, unmanaged second-growth, and extended rotation Pinus resinosa forests in Minnesota, USA. Forest Ecology and Management, 291: 110-118. doi: 10.1016/j.foreco.2012.11.033

Solís, R., Aguirre Calderón, O. A., Treviño, E. J., Jiménez, J., Jurado, E., \& Corral, J. (2006). Efecto de dos tratamientos silvícolas en la estructura de ecosistemas forestales en Durango, México. Madera y Bosques, 12(2), 49-64. doi: $10.21829 /$ myb.2006.1221242

Unión de Comunidades Forestales Zapotecas-Chinantecas [UZACHI]. (2003). Programa de Manejo Forestal para el predio de Capulálpam de Méndez, Municipio del mismo nombre (2003-2013). Oaxaca, México: Dirección Técnica Forestal.

Valappil, N. I. (1997). A physiologically based comparison of even- and multi-aged ponderosa pine stand productivity. Tesis de doctorado. University of Montana, Missoula, USA.

Vilá, A., Martínez, J., Vayreda, J., \& Retana, J. (2011). Structural and climatic determinants of demographic rates of Scots pine forest across the Iberian Peninsula. Ecological Society of America, 1(4), 1162-1172. doi: 10.1890/10-0647.1

Manuscrito recibido el 9 de julio de 2018

Aceptado el 25 de febrero de 2019

Publicado el 13 de noviembre 2019

Este documento se debe citar como:

Ramírez S., R., Ángeles P., G., Hernández R., P., Cetina A., V. M., Plascencia E., O., \& Clark-Tapia, R. (2019). Efectos del aprovechamiento forestal en la estructura, diversidad y dinámica de rodales mixtos en la Sierra Juárez de Oaxaca, México. Madera y Bosques, 25(3), e 2531818. doi: 10.21829/myb.2019.2531818

Madera y Bosques por Instituto de Ecología, A.C. se distribuye bajo una Licencia Creative Commons Atribución-NoComercialCompartirlgual 4.0 Internacional. 